\title{
Editorial: Sustainable Production of Bioactive Pigments
}

\author{
Wee Sim Choo ${ }^{1 *}$, Laurent Dufossé ${ }^{2 *}$ and Lourdes Morales-Oyervides ${ }^{3 *}$ \\ ${ }^{1}$ School of Science, Monash University Malaysia, Subang Jaya, Malaysia, ${ }^{2}$ ESIROI Agroalimentaire, Université de la Réunion, \\ Saint-Denis, France, ${ }^{3}$ Department of Chemical Engineering, Universidad Autónoma de Coahuila, Saltillo, Mexico
}

Keywords: anthocyanin, bacterial pigments, carotenoid, food waste, fungal pigments, green technology, marine sponge, microalgae

\section{Editorial on the Research Topic}

\section{Sustainable Production of Bioactive Pigments}

OPEN ACCESS

Edited by:

Ashish Rawson,

Indian Institute of Food Processing

Technology, India

Reviewed by:

Sergiy Smetana,

German Institute of Food

Technologies, Germany

${ }^{*}$ Correspondence:

Wee Sim Choo

choo.wee.sim@monash.edu

Laurent Dufossé

laurent.dufosse@univ-reunion.fr

Lourdes Morales-Oyervides

lourdesmorales@uadec.edu.mx

Specialty section:

This article was submitted to

Sustainable Food Processing,

a section of the journal

Frontiers in Sustainable Food Systems

Received: 01 March 2021

Accepted: 18 March 2021

Published: 15 April 2021

Citation:

Choo WS, Dufossé $L$ and

Morales-Oyervides L (2021) Editorial:

Sustainable Production of Bioactive

Pigments.

Front. Sustain. Food Syst. 5:674311.

doi: 10.3389/fsufs.2021.674311
Pigments are compounds that are perceived by humans to have color. Natural pigments such as chlorophylls are the source of green in all plants and the most abundant pigments in nature. Natural pigments are also responsible for yellow, orange, red, blue, and purple colors found in nature (Choo, 2019). Anthocyanins, carotenoids, and betalains are the common plant pigments found in nature besides chlorophyll (Choo, 2018). Besides plant pigments, pigments are derived to a lesser extent from insects or animals. Emerging sources of pigments are from microalgae and microorganisms (Morales-Oyervides et al., 2020). Pigments are considered to be bioactive if they have an interaction with, or have an effect on, cell tissue in the human body. There is huge interest in bioactive pigments that render beneficial health effects on humans. Consumers are driving the demand for natural food compounds such as bioactive pigments. However, continual prospecting of terrestrial or aquatic natural resources for natural food compounds will impose a huge demand on these resources. Sustainable production of bioactive pigments is therefore needed to ensure continuity of use for future generations and allow feasible increase in the utilization of these bioactive pigments.

This Research Topic provides reviews and researches on the sustainable production of various bioactive pigments. This Research Topic comprises a collection of 12 articles, including 4 reviews, 2 mini-reviews, and 6 original research articles. The review by Yong et al. provides a comprehensive evaluation of the impact of high-pressure homogenization $(\mathrm{HPH})$ on the extractability and stability of phytochemicals such as anthocyanins, carotenoids, and others. HPH technology is considered as green technology as it does not use polluting solvents, short processing duration, low consumption of energy, and emission of carbon dioxide (Mesa et al., 2020). Another review by Venil et al. highlights the significance of bacterial colorants and summarizes their application in food and pharma industries. The potential of bacterial pigments for mass production of diversified coloring properties was first prospective and is now getting the notable importance and attention of both the researchers and industries. Tactics in strain improvement, fermentation conditions, metabolic engineering, and easy extraction techniques are needed to produce high-end products. Likewise, Rana et al. reviewed the types of microbial pigments used as food additives; the authors emphasized the colorants associated biological activities, recent advancements and future challenges. Lopes and Ligabue-Braun summarized the information obtained during the last decade regarding the valorization of agro-industrial residues as an eco-friendly and low-cost alternative for the production of microbial pigments. Certainly, natural bioactive pigments are molecules with high added-value, which could be key to develop cost-effective processes using wastes toward a sustainable future.

The two mini-reviews investigate other aspects of microbial pigments. Pailliè-Jiménez et al. state that microorganisms, because of their genetic simplicity as compared with plants, may be a better 
source to understand biosynthetic mechanisms and to be engineered for producing high pigment yields. Moreover, despite the origin of the pigmented microorganism, it seems very important to develop protocols using organic industrial residues and agricultural by-products as substrates for pigment production and find novel green strategies for rapid pigment extraction. Despite the huge concern in secluding and collecting marine bacteria, microbial metabolites are progressively alluring to science due to their wide-ranging applications in various fields, particularly those with distinctive color pigments. The mini-review by Velmurugan et al. is an appraisal of the studies undertaken over the past 5 years on the bacterial pigments sourced from the marine environment.

The original research section starts with De Wit et al. who report the antioxidant properties of fresh and processed cactus pear fruit peels of different fruit-colored cultivars. Cactus pear fruit peels are usually discarded and considered as waste. In this work, authors found that the purple and orange-colored cultivars were the best choice in terms of antioxidant properties. Coffee (Coffea arabica L.) by-products are another source of carotenoids and phenolic compounds investigated by Esquivel et al.. Analysis of pulp and peels of Arabica coffee varieties with different external fruit color allowed the identification of a great number of compounds using high-performance liquid chromatography with diode array detection and electrospray ionization multistage mass spectrometry (HPLC-DAD-ESI-MSn).

Marine sponges have been known to harbor microorganisms that produce secondary metabolites like bioactive pigments. One red bacterium isolated from the sponge Petrosia sp. in Hawai'i by Sakai-Kawada et al. was identified as belonging to the Pseudoalteromonas genus. The draft genome was sequenced, assembled, and annotated. This revealed a prodiginine biosynthetic pathway and the first cited-incidence of a prodiginine-producing Pseudoalteromonas species isolated from a marine sponge host. Further, it has been well-documented that the fungal pigments produced by Monascus strains possess

\section{REFERENCES}

Choo, W. S. (2018). "Betalains: Application in functional foods" in Bioactive Molecules in Food. Reference Series in Phytochemistry, eds J. M. Mérillon, and K. G. Ramawat (Cham: Springer International Publishing), 1-28.

Choo, W. S. (2019). "Fruit pigment changes during ripening", in Encyclopedia of Food Chemistry, eds P. Varelis, L. Melton, and F. Shahidi (Amsterdam: Elsevier), 117-123.

Mesa, J., Hinestroza-Cordoba, L. I., Barrera, C., Segu, L., Betoret, E., and Betoret, N. (2020). High homogenization pressures to improve food quality, functionality and sustainability. Molecules 25:3305. doi: 10.3390/molecules251 43305

Morales-Oyervides, L., Ruiz-Sánchez, J. P., Oliveira, J. C., Sousa-Gallagher, M. J., Méndez-Zavala, A., Giuffrida, D., et al. (2020). Biotechnological approaches various biological activities. Accordingly, Mohankumari et al. investigated the effect of Monascus fermented rice (RMR, traditional Chinese food additive) on the cholesterol blood levels in rats fed with a high-fat diet. The authors reported satisfactory results concluding that RMR is a promising anti-atherogenic food supplement. Other biological activities displayed by the natural bioactive pigments which have called researchers attention are the antimicrobial properties. In this regard, Mekala et al. isolated and characterized a pigment producer bacterium (Exiguobacterium sp GM010) which pigments showed bactericidal effects against food-borne pathogens, both Gram positive and Gram negative. The antibacterial mode of action and non-toxic effect of the pigments toward A. franciscana were elucidated, suggesting the potential application of the pigments in food preservation. Indeed, the properties of the bioactive pigments make them promising for a wide range of industrial applications. However, one important aspect of ensuring any industrial application is the stability of the pigments, especially if they are compared with synthetic pigments. On this subject, Ghosh and Mishra studied the stability of C-phycoerythrin, a water-soluble pigment, which was produced by the microalgae Lyngbya sp. (CCNM 2053). The pigments were stable under acidic to neutral conditions ( $\mathrm{pH} 3-8)$ and presented moderate stability under light exposure; however, the pigments were poorly stable under high temperatures $\left(>50^{\circ} \mathrm{C}\right)$.

This editorial summarizes the articles in this Research Topic. We hope that this collection of articles will contribute to the advancement of research on the sustainable production of bioactive pigments. Lastly, we would like to thank all authors, reviewers and Frontiers editorial team for their contributions in this Research Topic.

\section{AUTHOR CONTRIBUTIONS}

All authors listed have made a substantial, direct and intellectual contribution to the work, and approved it for publication.

for the production of natural colorants by Talaromyces/Penicillium: a review. Biotechnol. Adv. 43:107601. doi: 10.1016/j.biotechadv.2020.107601

Conflict of Interest: The authors declare that the research was conducted in the absence of any commercial or financial relationships that could be construed as a potential conflict of interest.

Copyright (c) 2021 Choo, Dufossé and Morales-Oyervides. This is an open-access article distributed under the terms of the Creative Commons Attribution License (CC BY). The use, distribution or reproduction in other forums is permitted, provided the original author(s) and the copyright owner(s) are credited and that the original publication in this journal is cited, in accordance with accepted academic practice. No use, distribution or reproduction is permitted which does not comply with these terms. 